\title{
The Cosmic Radius of Observable Universe
}

\author{
Xiaoyun Li, Suoang Longzhou, La Ba Sakya Genzon \\ School of Science and Engineering, The Chinese University of Hong Kong, Shenzhen, China \\ Email: lixiaoyun@cuhk.edu.cn, suoanglongzhou@cuhk.edu.cn, sakyagenzon@cuhk.edu.cn
}

How to cite this paper: Li, X.Y., Longzhou, S. and Genzon, L.B.S. (2022) The Cosmic Radius of Observable Universe. Journal of High Energy Physics, Gravitation and Cosmology, 8, 1-13.

https://doi.org/10.4236/jhepgc.2022.81001

Received: September 23, 2021

Accepted: November 15, 2021

Published: November 18, 2021

Copyright (c) 2022 by author(s) and Scientific Research Publishing Inc. This work is licensed under the Creative Commons Attribution International License (CC BY 4.0).

http://creativecommons.org/licenses/by/4.0/

\begin{abstract}
This paper introduces three cosmic expansion models with constant, decelerating and accelerating speed of expansion respectively. Then characters of these cosmic expansion models are compared. Based on these cosmic expansion models, the thresholds of observable universe are calculated via simulations, where the earliest observable cosmic radius $R\left(t_{\text {earliest }}\right)$ is always $0.368 R$ ( $R$ is cosmic radius at current universe time) for any cosmic expansion models.
\end{abstract}

\section{Keywords}

Observable Universe, Threshold, Simulation, Cosmic Expansion Model

\section{Introduction and Related Works}

The measurement of the distance of stars and galaxies in the universe has always been one of the main research topics of cosmology. About 100 years ago, astronomers discovered that the light of distant stars has a redshift, so it is speculated that these stars are moving away from the earth. After that, scientists used Einstein's field equations to introduce the universe's space-time expansion model [1]. Later, astronomers discovered that stars and galaxies farther away from the earth have larger separation speed. Hence the stellar distance according to its redshift is calculated based on Hubble's law [2].

In physical cosmology, the cosmic expansion model is established via Einstein's field equations, to constrain the metric of an isotropic uniform universe using Robertson-Walker metrics [1], and perform the derivation of universe evolution dynamics. Then there is a first-order differential equation of spatial scale $R(t): \dot{R}^{2}+k$. When $k>0$, the cosmic expansion decelerates; when $k<0$, the cosmic expansion accelerates; when $k=0$, the universe expands at a constant speed [3].

Based on above universe expansion model, the Big Bang theory describes how 
the universe expanded from an initial state of extremely high density and high temperature [4]. The Big Bang theory is compatible with Hubble's law. Hubble's law [2] is the observation in physical cosmology that stars are moving away from the Earth at speeds proportional to their distance.

The photon epoch was the period in the evolution of the early universe in which photons dominated the universe. The photon epoch started at about 10 seconds after the Big Bang, and ended at 370,000 years after the Big Bang, when the temperature of the universe fell so that photons no longer interacted frequently with matter including Atomic nuclei and electrons [5].

In [6], it mentioned a so-called horizon problem. Since the known cosmic space-time is almost isotropy and homogenous with almost evenly distributed cosmic energy density, if the speed of light $c$ is a constant, which is slower than the speed of cosmic expansion, then the energy-carrying light could not reach the universe's boundaries quickly enough, resulting in a significant difference of measurable cosmic energy density.

To solve the horizon problem, a hypothesis is made that the speed of light in early universe is much faster than the speed of light $c$ in current universe. This hypothesis is proved by a certain fixed value of spectral index, which describes the initial density ripples in the Universe [6]. The latest spectral index figure reported by Planck satellite [7] verified the spectral index value calculated by [6] very well, with an error less than $1 \%$.

Based on the above cosmic expansion dynamics using Robertson-Walker metrics [1], and the assumption that the speed of light equals the speed of cosmic expansion, this paper introduces three cosmic expansion models with constant, decelerated and accelerated speed of expansion respectively. And the characters of these models are compared. Then the threshold of observable cosmic space-time is derived. Note that, this paper is not to judge which cosmic expansion model is correct, but to find out the threshold of observable cosmic space-time.

\section{List of Terms}

T: The current universe time $T=13.82$ Gyrs.

$t$. Universe age or time, where $0 \leq t \leq T$.

$c$. The constant speed of light.

$R$ : Universe radius at $T$.

$M$ : Total mass of the universe.

$G$ : Gravitational constant.

$\Phi_{G}$ : Gravitational potential.

$\Phi_{G}(t)$ : Gravitational potential at universe time $t$.

$V_{G}$ : Velocity of propagation of gravitation.

$V_{\text {Expan }}(t)$ : Speed of cosmic expansion at $t$.

$R(t)$ : Universe radius at $t$.

$R(0)$ : Initial Universe radius at $t=0$.

$d$ : Light source. 
$O$ : The Observer and the center of the cosmic horizon.

$v(t)$ : Separation Speed at time $t$.

$S(t)$ : The distance between $O$ and the beam of light $(B)$ emitted by $d$.

$S$ : The distance of $d$ observed by $O$.

Gyr: Giga years.

Gly: Giga light years.

$t_{\text {earliest }}$ : The earliest observable universe time.

$R\left(t_{\text {earliest }}\right)$ : The cosmic radius at the earliest observable universe time $t_{\text {earliest }}$.

$S_{\text {furthest }}:$ THE furthest observable distance.

$r_{\text {expan }}(t)$ : Cosmic expansion rate at $t$.

$r_{\text {acc }}(t)$ : Cosmic acceleration rate at $t$.

$r(t):$ Spatial scale at $t$.

$\Delta t:$ Unit time.

\section{Cosmic Expansion Models}

\subsection{Constant Cosmic Expansion Model}

Assume the speed of cosmic expansion $V_{\text {Expan }}=c$, where $c$ is the constant speed of light as shown in Figure 1. The universe age is $T=13.82$ Gyrs according to [7]. Therefore the current cosmic radius $R=c T=13.82$ Glys , assuming $R(t=0) \rightarrow 0$. The universe radius $R(t)$ during the cosmic expansion is a straight line as shown in Figure 2.

The constant cosmic expansion model is apparently contradicted to the Big Bang model. Since it has no inflation period in early universe. But this simplified cosmic expansion model could be a benchmark which will help us to understand the cosmic expansion and the observable universe easier [8].



Figure 1. The speed of cosmic expansion for decelerated and accelerated models. 


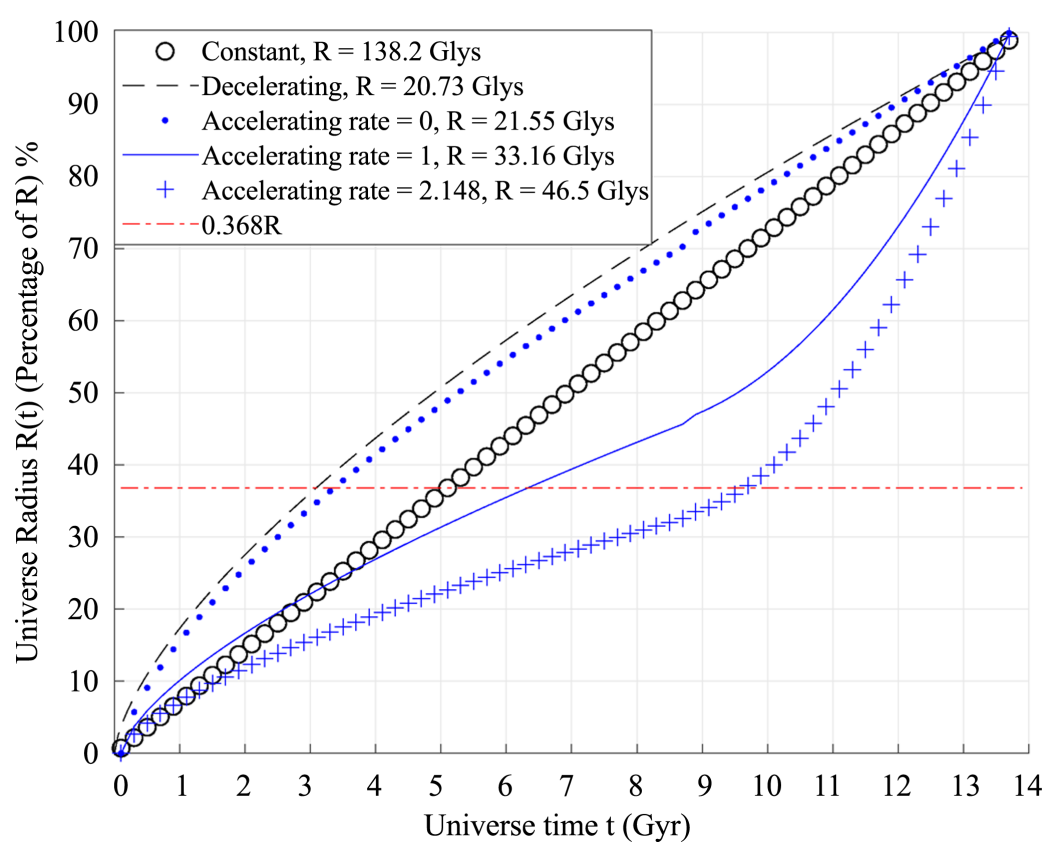

Figure 2. Universe Radius for different cosmic expansion models.

\subsection{Decelerated Cosmic Expansion Model}

The speed of light is the constant $c . V_{\text {expan }}(t)$ is cosmic expansion speed at universe time $t$, which is the increasing speed of cosmic radius $R(t) \cdot r_{\text {expan }}(t)$ is cosmic expansion rate at universe time $t$, where $r_{\text {expan }}(t)=V_{\text {expan }}(t) / c$.

The propagation speed of light equals the propagation speed of electromagnetic field $c$. On the other hand, light also has the characters of photon gas. Therefore, during cosmic spatial expansion, photon gas (light) also expands at the same way. Therefore it is reasonable to assume that, the speed of light considering cosmic expansion rate is $c(t)=r_{\text {expan }}(t) c=V_{\text {expan }}(t)$, such that the speed of light equals the speed of cosmic expansion, in order to avoid the horizon problem.

The spatial scale $r(t)=r_{\text {expan }}(t) c \Delta t$. Let time unit $\Delta t$ equals constant $1 / c$. Therefore the spatial scale $r(t)=r_{\text {expan }}(t)$. Hence the speed of light observed by observer at any universe time $t$ equals $\frac{r_{\text {expan }}(t) c}{r(t)}=\frac{r_{\text {expan }}(t) c}{r_{\text {expan }}(t)}=c$. It shows that, both spatial scale and the speed of light are varied according to cosmic expansion rate $r_{\text {expan }}(t)$, therefore the speed of light at any universe time $t$ is the dimensionless constant $c$.

Since the spatial scale $r(t)=r_{\text {expan }}(t)$ varied with cosmic expansion rate at different universe time $t$, in order to present all kinds of distances with a uniform spatial scale $r$, let $r=1$. Then for any universe time $t$ with spatial scale $r(t)=r_{\text {expan }}(t)$, any distance $d$ is presented as $r_{\text {expan }}(t) d$ using the uniform spatial scale $r=1$. The uniform spatial scale $r=1$ is used in equations of decelerating or accelerating cosmic expansion models in the following sections. Then, 


$$
\begin{gathered}
\Phi_{G}=\frac{G M}{R}=c^{2} \\
\rightarrow \frac{G M}{R(t)}=r_{\text {expan }}(t)^{2} c^{2} \\
\rightarrow \frac{R(t)}{R}=\frac{1}{r_{\text {expan }}(t)^{2}} \\
\rightarrow V_{\text {Expan }}(t)=r_{\text {expan }}(t) c=c \sqrt{\frac{R}{R(t)}}
\end{gathered}
$$

The derivation of the cosmic decelerating model is based on Equation (1) [9]. The proof of Equation (1) is provided in Appendix of this paper. Note that, the purpose of this paper is not to prove the correctness of any cosmic expansion model.

According to Equation (1), then Equations (2) and (3) can be derived as follows, where $G$ is Gravitational constant, $M$ is the cosmic total mass which is assumed to be a constant too.

According to [7], take universe age $T=13.82$ Gyrs, which is the current universe time. The initial universe radius $R\left(t_{0}\right)$ is set to a random small value. Let $R\left(t_{0}\right)=10^{10} \mathrm{~m}$, where $t_{0}=0 . R\left(t_{0}\right)$ can be any random value less than $10^{10} \mathrm{~m}$, which does not affect the simulation results, because the time period it takes for the cosmic radius to expand to $10^{14} \mathrm{~m}$ is less than 1 second. The current universe radius $R$ is temporarily set to 46.5 Glys according to [10]. Hence the initial conditions for simulation are as follows.

$$
\begin{gathered}
R\left(t_{0}\right)=R(0)=10^{10} \mathrm{~m} \\
T=13.82 \text { Gyrs } \\
R=46.5 \text { Glys }
\end{gathered}
$$

According to Equation (3), the initial speed of cosmic expansion $V_{\text {Expan }}\left(t_{0}\right)$ can be calculated as follows.

$$
V_{\text {Expan }}\left(t_{0}\right)=V_{\text {Expan }}(0)=c \sqrt{\frac{R}{R(0)}}
$$

According to Equation (3), after time period $d t$, there are:

$$
\begin{gathered}
R(d t)=R(0)+V_{\text {Expan }}(0) d t \\
R(t+d t)=R(t)+V_{\text {Expan }}(t) d t
\end{gathered}
$$

Simulation were performed according to the above procedure. Simulation results show that, when $R(t)=46.5$ Glys, $t>T$. Only when $R(t)=20.73$ Glys, $t=T=13.82$ Gyrs. For $t=10$ million years and $t=100$ million years, the speed of cosmic expansion is around $11.1 c$ and $5.2 c$ respectively for the decelerating model as showing in Figure 1.

Figure 3 shows the process that the speed of cosmic expansion decreased from $2400 c$ to $c$, during the period of universe time 1 year $\leq t \leq 13.82$ Gyrs . 


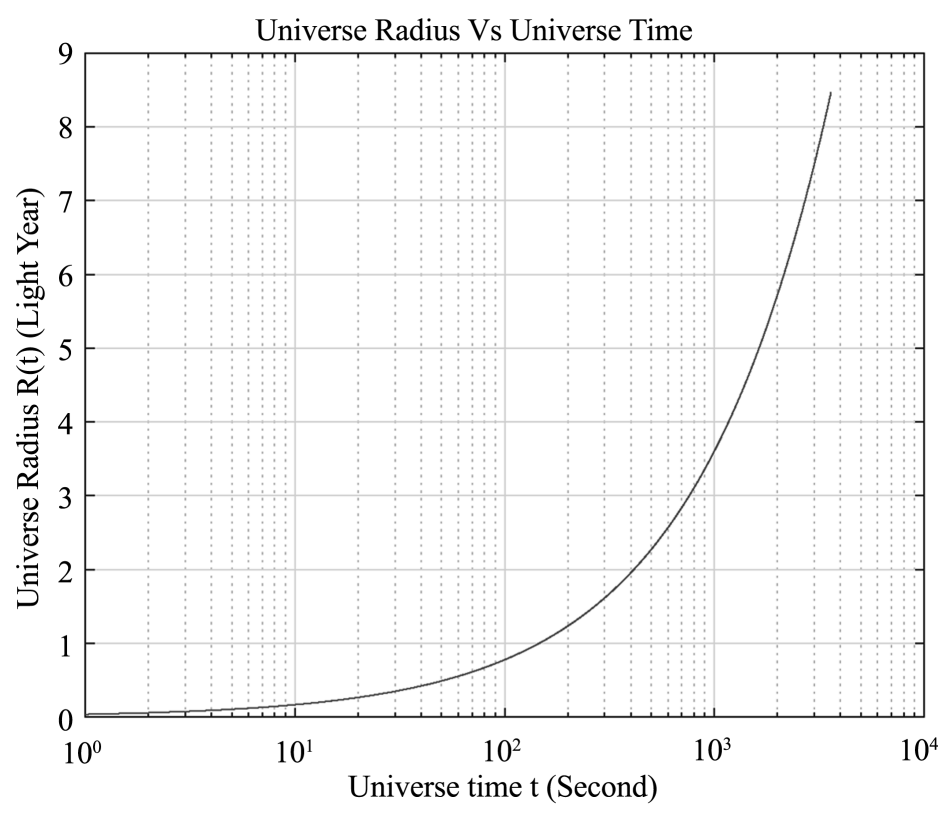

Figure 3. Cosmic radius for $t \geq 1$ year (decelerating model).

The speed of cosmic expansion $V_{\text {Expan }}(t)$ decreased to less than $100 c$ for $t=1$ million years. And $V_{\text {Expan }}(t)$ decreased less than $2.5 \%$ in the last 1 Gyrs of universe time.

The simulation of cosmic decelerating model also mimics the inflation of early universe very well as shown in Figure 4. It shows that the cosmic radius $R(t)$ expanded from the initial size $R\left(t_{0}\right)=10^{10} \mathrm{~m}$ to 1 light year in less than 2 minutes of universe time, and $R(t)$ expanded to more than 8 light years when universe time $t=1$ hour.

\subsection{Accelerated Cosmic Expansion Model}

According to the Big Bang theory and the accelerating cosmic expansion model, the cosmic expansion decelerated after the Big Bang until universe time $t=9$ Gyrs . Then the acceleration of cosmic expansion began after $t=9$ Gyrs (4.82 Gyrs ago) [11].

Since the above decelerating cosmic expansion model can mimic the inflation of early universe very well, and the current cosmic radius $R=20.73$ Glys in the decelerating model, which is much less than the current cosmic radius of 46.5 Glys in the accelerating model [10], therefore it is reasonable to assume that in the accelerating model, the cosmic expansion procedure is the same as that in the decelerating model for $t \leq 9$ Gyrs as shown in Figure 1 .

Figure 1 shows that the speed of cosmic expansion decreased from $11.1 \mathrm{c}$ (when $t=0.01 \mathrm{Gyrs}$ ) to $1.24 c$ at $t=9$ Gyrs in the decelerating model. While in the accelerating model, assume the speed of cosmic expansion increase linearly for $t>9$ Gyrs depending on different acceleration rate $r_{a c c}(t)$, and the speed of cosmic expansion for accelerating model is exactly the same as the decelerating model for $t \leq 9$ Gyrs . 


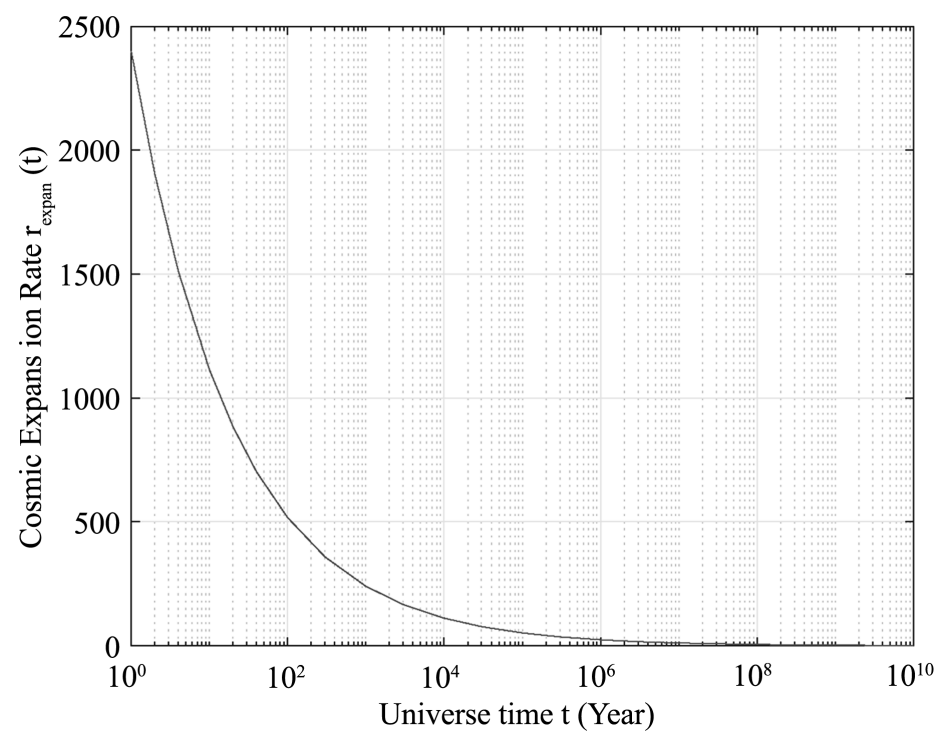

Figure 4. Cosmic expansion rate for $t \leq 1$ hour (decelerating model).

If $r_{\text {acc }}(t)=0$, the speed of cosmic expansion $r_{\text {expan }}(t)$ is fixed on 1.24 for $t>9$ Gyrs .

If $r_{a c c}(t)=1$, where $r_{\text {expan }}(t)$ increases by 1 per Gyrs, the cosmic expansion rate $r_{\text {expan }}(t)$ increases linearly from 1.24 (at $t=9$ Gyrs) to 5.95 at current universe time $T$.

If $r_{a c c}(t)=2.148$ where $r_{\text {expan }}(t)$ increases by 2.148 per Gyrs, the cosmic expansion rate increases linearly from 1.24 (at $t=9$ Gyrs) to 11.46 at $T$, and cosmic radius $R$ expands to 46.5 Glys.

\section{Deriving the Threshold of Observable Universe}

\subsection{Constant Cosmic Expansion Model}

In the constant cosmic expansion model, the cosmic radius $R(t=0) \rightarrow 0$, and the cosmic radius $R(t)$ expands by the speed of cosmic expansion $V_{\text {Expan }}=c$. Therefore, for a given universe time $t \leq T$, then $R(t)=t \times c$.

If there is a light source $d$ at boundary of the universe, $d$ emitted a beam of light $(B)$ to the Observer $O(O$ is the earth, which is the center of cosmic horizon) at time $t$ as shown in Figure 5. Then at the position of $d$ within universe time $t$, the earth $(O)$ has a separation speed $v(t)=c$ because the cosmic expansion speed $V_{\text {Expan }}=c$, assuming the cosmic space-time is isotropy and homogenous [8].

The distance between the beam of light $(B)$ and the earth $(O)$ is $S(t)=R(t)$ at universe time $t$. After time period $d t$, the beam of light $(B)$ approached $O$ by speed of light $c$. Because the cosmic expansion speed is constant $c$, therefore the separation speed of the earth $(O)$ is $v(t+d t)=c \frac{S(t)}{R(t+d t)}$, assuming the cosmic space-time is isotropy and homogenous, and the separation speed is proportional to the distance according to Hubble's law [2]. Hence, 


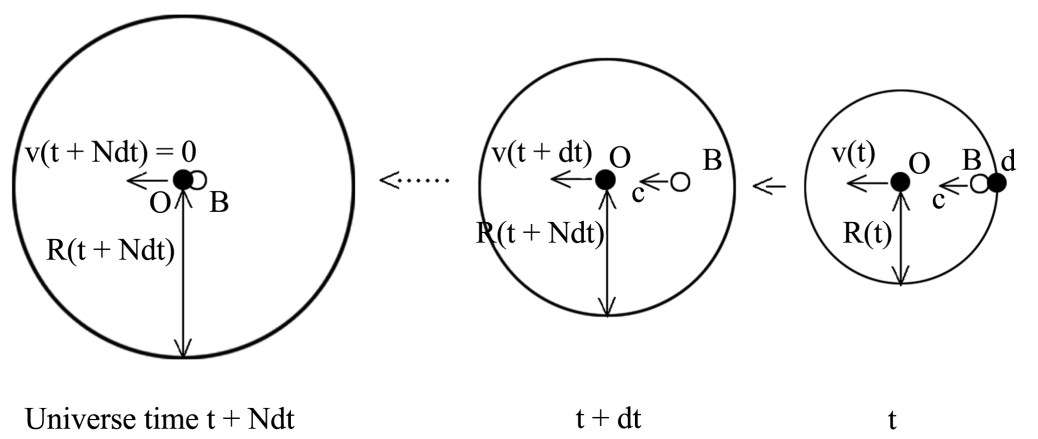

Figure 5. Observing the light source $d$ during cosmic expansion.

$$
\begin{gathered}
R(t)=c \times t \\
v(t)=c \\
S(t)=R(t)=c \times t \\
\rightarrow R(t+d t)=c \times(t+d t) \\
v(t+d t)=c \frac{S(t)}{R(t+d t)} \\
S(t+d t)=R(t)-c \times d t+v(t+d t) d t
\end{gathered}
$$

At universe time $t+N d t$ where $N d t=N \times d t$, then

$$
\begin{gathered}
\rightarrow R(t+N d t)=c \times(t+N d t) \\
v(t+N d t)=c \frac{S[t+(N-1) d t]}{R(t+N d t)} \\
S(t+N d t)=R(t)-c \times N d t+d t \sum_{K=1}^{N} v(t+K d t)
\end{gathered}
$$

If $t+N d t=T$, and $S(t+N d t)=0$, then the separation speed $v(t+N d t)=0$, and the beam of light $(B)$ has arrived the earth $(O)$ exactly at current universe time $T$, as shown in Figure 5 and Figure 6 [8].

Simulation by Matlab is performed according to the above pseudo code. The simulation results are as follows.

$$
t_{\text {earliest }}=5.084 \text { Gyrs }=0.368 T \text {, }
$$

where

$$
\begin{gathered}
T=13.82 \text { Gyrs } \\
R\left(t_{\text {earliest }}\right)=5.084 \text { Glys }=0.368 R
\end{gathered}
$$

The above simulation results show that, at universe time $t_{\text {earliest }}=0.368 T$, universe radius $R\left(t_{\text {earliest }}\right)=0.368 R$ (where $R=13.82$ Glys), the light source $d$ at boundary of the universe has emitted a beam of light $B$ towards the center of cosmic horizon $O$ (the earth). After time period $T-t_{\text {earliest }}, B$ has arrived $O$ exactly at universe time $T$. Therefore the light source $d$ at boundary of the universe at $t_{\text {earliest }}$ is observed by observer $O$ right now at universe time $T$ [8], as shown in Figure 2. 


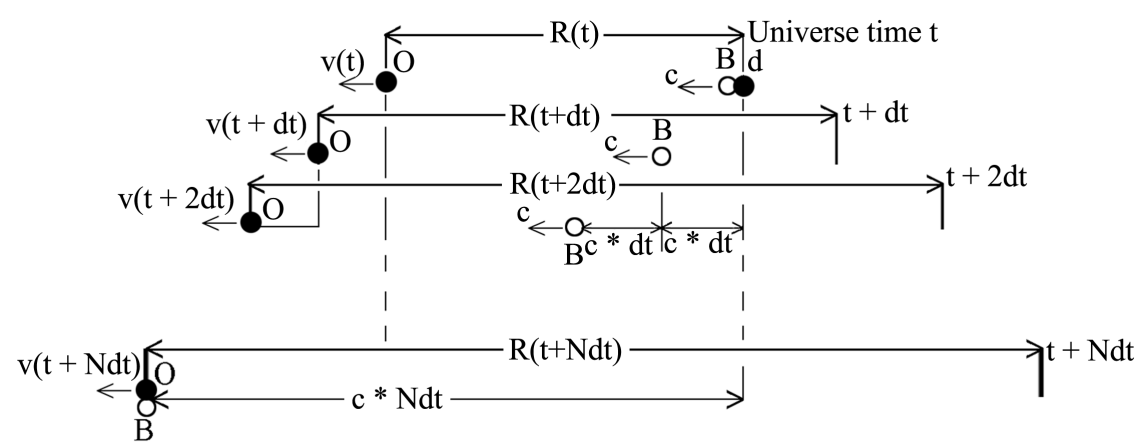

Figure 6. The procedure to observe the light source $d$ during cosmic expansion.

If the light source $d$ has emitted another beam of light $B^{\prime}$ at time $t_{\text {earliest }}$ to the opposite direction of $B$, then when the beam of light $B$ arrives $O$ (the center of the cosmic horizon) at universe time $T, B^{\prime}$ also arrives the boundary of the universe with radius $R$, because the speed of light equals to the speed of cosmic expansion. Therefore the distance $S$ of light source $d$ as observed by $O$ is the distance traveled by the beam of light $B$ (or $B$ ) during time period $T-t_{\text {earliest }}$. Therefore,

$$
S_{\text {furthest }}=R-R\left(t_{\text {earliest }}\right)=0.632 R
$$

If $d$ is within the boundary of the universe at time $t^{\prime}<t_{\text {earliest }}$, then $R\left(t^{\prime}\right)<R\left(t_{\text {earliest }}\right)$, therefore the beam of light emitted by $d^{\prime}$ has arrived $O$ at universe time $T^{\prime}<T$, therefore $d^{\prime}$ can be observed by $O$ at $T^{\prime}$, which was at time period $T-T^{\prime}$ ago. Hence time $t_{\text {earliest }}$ is the earliest observable universe time when signals emitted by a light source $d$ at that time can be received by $O$ at current universe time $T, S_{\text {furthest }}=R-R\left(t_{\text {earliest }}\right)=0.632 R$ is the furthest observable distance from where a light source $d$ can be observed by $O$ at current universe time $T$ [8].

\subsection{Decelerated Cosmic Expansion Model}

In the decelerating cosmic expansion model, for any universe time $t$, universe radius $R(t)$ can be calculated by the simulation as described in the last section, as shown in Figure 2, and the cosmic expansion speed $V_{\text {Expan }}(t)=c(t)=r_{\text {expan }}(t) c$ as described in the last section (Subsection 2.2).

Similar to the above constant expansion model, for a given universe time $t<T$, there is a light source $d$ at the boundary of the universe with cosmic radius $R(t)$. And $d$ emitted a beam of light $(B)$ towards the observer $O$ at time $t$ as shown in Figure 5 and Figure 6, where the speed of light $c$ is replaced by $r_{\text {expan }}(t) c$ in the decelerating model. Then the following equations can be derived.

$$
\begin{gathered}
r_{\text {expan }}(t)=\sqrt{\frac{R}{R(t)}} \\
v(t)=r_{\text {expan }}(t) c
\end{gathered}
$$




$$
\begin{gathered}
S(t)=R(t) \\
\rightarrow r_{\text {expan }}(t+d t)=\sqrt{\frac{R}{R(t+d t)}} \\
v(t+d t)=r_{\text {expan }}(t+d t) c \frac{S(t)}{R(t+d t)} \\
S(t+d t)=R(t)-r_{\text {expan }}(t+d t) c d t+v(t+d t) d t
\end{gathered}
$$

At universe time $t+N d t$, then,

$$
\begin{gathered}
\rightarrow r_{\text {expan }}(t+N d t)=\sqrt{\frac{R}{R(t+N d t)}} \\
v(t+N d t)=r_{\text {expan }}(t+N d t) c \frac{S[t+(N-1) d t]}{R(t+N d t)} \\
S(t+N d t)=R(t)-d t \sum_{K=1}^{N} r_{\text {expan }}(t+K d t) c+d t \sum_{K=1}^{N} v(t+K d t)
\end{gathered}
$$

If $t+N d t=T$, and $S(t+N d t)=0$, then the separation speed $v(t+N d t)=0$, and the beam of light $(B)$ has arrived the earth $(O)$ exactly at current universe time $T$, as shown in Figure 5 and Figure 6.

Simulation is performed according to the above process. The simulation results are as follows.

$$
t_{\text {earliest }}=3.083 \text { Gyrs }=0.223 T \text {, }
$$

where

$$
\begin{gathered}
T=13.82 \text { Gyrs } \\
R\left(t_{\text {earliest }}\right)=7.626 \text { Glys }=0.368 R, \\
S_{\text {earliest }}=R-R\left(t_{\text {earliest }}\right)=0.632 R
\end{gathered}
$$

The above simulation results show that, in the decelerating expansion model, at the earliest observable universe time $t=3.083 \mathrm{Gyrs}$ with universe radius $R\left(t_{\text {earliest }}\right)=0.368 R$, a light source $d$ at boundary of the universe can be observed by the observer $O$ at universe time $T$ with the furthest observable distance $S_{\text {earliest }}=0.632 R$, where $R=20.73$ Glys .

\subsection{Accelerated Cosmic Expansion Model}

In the accelerating cosmic expansion model, it is said that the cosmic expansion is decelerated after the Big Bang until universe time $t=9 \mathrm{Gyrs}$, and the cosmic expansion was accelerating when universe time $t>9$ Gyrs until now with current universe time $T=13.82$ Gyrs [11].

Since the decelerating cosmic expansion model can mimic the inflation of early universe very well, therefore it is assumed that in the accelerating model, the speed of cosmic expansion is exactly the same as that in the decelerating model for $t \leq 9$ Gyrs as shown in Figure 1 .

For $t>9$ Gyrs, the acceleration rate $r_{\text {acc }}(t)$ in the accelerating model is set 
to 0,1 and 2.148 respectively as mentioned in the last section (Subsection 2.3). simulation results are as follows, as shown in Figure 2.

For $r_{\text {acc }}(t)=0, t_{\text {earliest }}=3.27$ Gyrs,$R=21.55$ Glys,$R\left(t_{\text {earliest }}\right)=0.368 R$.

For $r_{\text {acc }}(t)=1, t_{\text {earliest }}=6.24$ Gyrs,$R=33.16$ Glys,$R\left(t_{\text {earliest }}\right)=0.368 R$.

For $r_{\text {acc }}(t)=2.148, t_{\text {earliest }}=9.75$ Gyrs,$R=46.5$ Glys,$R\left(t_{\text {earliest }}\right)=0.368 R$.

Simulation shows that, for any other acceleration rate $r_{\text {acc }}(t)>0$, and for the accelerating period begins at any universe time $t$ other than 9 Gyrs, the earliest observable cosmic radius $R\left(t_{\text {earliest }}\right)$ is always $0.368 R$, although $R$ and $t_{\text {earliest }}$ are varied according to different acceleration rate. And the furthest observable distance is $S_{\text {furthest }}=R-0.368 R=0.632 R$ for any acceleration rate.

\subsection{Summary}

In summary, the earliest observable cosmic radius $R\left(t_{\text {earliest }}\right)$ is always $0.368 R$ for any cosmic expansion models including the constant, decelerating and accelerating models. Therefore the furthest observable distance is always

$S_{\text {furthest }}=R-0.368 R=0.632 R$ for any cosmic expansion models.

The earliest observable times $t_{\text {earliest }}$ are 3.083 Gyrs and 5.084 Gyrs for the decelerating and constant models respectively. $t_{\text {earliest }}$ is more than 3.27 Gyrs for acceleration rate $r_{a c c}(t)>0$ in the acceleration model as shown in Figure 2 . Therefore the lowest threshold for the earliest observable time $t_{\text {earliest }}$ is 3.083 Gyrs for any cosmic expansion model.

\section{Conclusion}

This paper introduces three cosmic expansion models with constant, decelerating and accelerating speed of expansion respectively. Then characters of these cosmic expansion models are compared. Based on these cosmic expansion models, the thresholds of observable universe are calculated via simulations, where the earliest observable cosmic radius $R\left(t_{\text {earliest }}\right)$ is always $0.368 R$ ( $R$ is cosmic radius at current universe time) for any cosmic expansion model, and the lowest threshold for the earliest observable time $t_{\text {earliest }}$ is 3.083 Gyrs for any cosmic expansion model.

\section{Conflicts of Interest}

The authors declare no conflicts of interest regarding the publication of this paper.

\section{References}

[1] Bergström, L. and Goobar, A. (2006) Cosmology and Particle Astrophysics. 2nd ed., Springer, Berlin.

[2] Hubble, E. (1929) A Relation between Distance and Radial Velocity among ExtraGalactic Nebulae. Proceedings of the National Academy of Sciences, 15, 168-173. https://doi.org/10.1073/pnas.15.3.168

[3] Hawking, S.W. and Ellis, G.F.R. (1973) The Large Scale Structure of Space-Time. Cambridge University Press, Cambridge. 
[4] NASA/WMAP Science Team (June 2011). Cosmology: The Study of the Universe. Universe 101: Big Bang Theory. Washington DC, NASA.

[5] Boesgaard, A.M. and Steigman, G. (1985) Big Bang Nucleosynthesis: Theories and Observations. Annual Review of Astronomy and Astrophysics, 23, 319-378. https://doi.org/10.1146/annurev.aa.23.090185.001535

[6] Afshordi, N. and Magueijo, J. (2016) The Critical Geometry of a Thermal Big Bang. Physical Review D, 94, Article ID: 101301(R). https://arxiv.org/pdf/1603.03312v2.pdf https://doi.org/10.1103/PhysRevD.94.101301

[7] Akrami, Y., Arroja, F., et al. (Planck Collaboration) (17 July 2018) Planck 2018 Results. I. Overview and the Cosmological Legacy of Planck. arXiv:1807.06205 [astro-ph.CO].

[8] Li, X.Y., Genzon, L.B.S., et al. (2021) The Observable Universe in a Simplified Cosmic Dynamic Model. Journal of Applied Mathematics and Physics, 9, 1322-1328. https://doi.org/10.4236/jamp.2021.96089

[9] Dai, Y.P., Dai, X.P. and Li, X.Y. (2015) The Relationship between the Speed of Light and Cosmic Background Potential. https://researchgate.net/publication/274712716

[10] Davis, T.M. and Lineweaver, C.H. (2004) Expanding Confusion: Common Misconceptions of Cosmological Horizons and the Superluminal Expansion of the Universe. Publications of the Astronomical Society of Australia, 21, No. 1, https://doi.org/10.1071/AS03040

[11] First Second of the Big Bang. How The Universe Works 3 (2014). Discovery Science.

[12] Landau, L.D. and Liftschitz, E.M. (1980) The Classical Theory of Fields. 4th Edition, Butterworth-Heinemann, Oxford. 


\section{Appendix}

In [12], it assumed that, the velocity of propagation of interactions (represented by $c$ ) is the same in all inertial systems of reference. In mathematically speaking, the velocity of propagation of interactions keeps invariant with the choice of different 4-dimensional space-time orthogonal coordinate system.

Similar to the assumption above, assume that the velocity of propagation of gravitation or interaction of material particles (represented by $V_{G}$ ) keeps invariant with the choice of different 4-dimensional space-time orthogonal coordinate system.

The cosmic gravitational potential $\Phi_{G}$ on cosmic sphere with universe radius $R$ is produced by all matter $M$ of the universe [9], where $\Phi_{G}=V_{G}^{2}$, and the gravitational potential energy of a material particle with mass $m$ is $E_{G}=m \Phi_{G}=m V_{G}^{2}$.

Then replace speed of light $c$ with $V_{G}$, the Lorenz transformation can be derived. And the equation of Lorentz Factor $\gamma$ is as follows [9].

$$
\gamma=\frac{1}{\sqrt{1-\frac{v^{2}}{V_{G}^{2}}}}
$$

Then based on Principle of Least Action, the Lagrangian of a material particle with mass $m$, and the relativistic Hamilton-Jacobi equation can be derived. Then the equations of electrodynamics, including Maxwell's equations, Lorentz force and d' Alembert's equation can be derived consequently [9] [12]. Hence it is proved that $V_{G}=c$, and $V_{G}^{2}=c^{2}=\Phi_{G}$. Then the following equation of gravitational potential can be derived.

$$
\Phi_{G}=\frac{G M}{R}=V_{G}^{2}=c^{2}
$$

${ }^{\star}$ End of Appendix ${ }^{\star}$ 